Revista Brasileira de Agricultura Irrigada v.9, no.4, p.218 - 224, 2015

ISSN 1982-7679 (On-line)

Fortaleza, CE, INOVAGRI - http://www.inovagri.org.br

DOI: $10.7127 /$ rbai.v9n400356

Protocolo 356.15 - 08/05/2015 Aprovado em 11/07/2015

\title{
DETERMINAÇÃO DO ÍNDICE DE ESTRESSE HÍDRICO EM TOMATEIROS CEREJA (Lycopersicum Solanum var. cerasiforme.) COM CÂMARA INFRAVERMELHA
}

\author{
Asdrúbal Jesús Farías Ramírez ${ }^{1}$, Rubens Duarte Coelho ${ }^{2}$, Maria Alejandra Moreno Pizani. ${ }^{3}$, \\ Cicero Jose da Silva ${ }^{4}$.
}

\begin{abstract}
RESUMO
A termometria infravermelha é uma possibilidade de detecção do estresse hídrico das plantas, através da medição da temperatura de uma porção do dossel vegetativo sem necessidade de contato físico com as folhas. Assim, o objetivo deste trabalho foi determinar o índice de estresse hídrico de tomateiros cereja através da temperatura foliar das plantas, com e sem estresse hídrico, cultivadas em casa de vegetação no Departamento de Engenharia de Biossistemas da Escola Superior de Agricultura "Luiz de Queiroz", em Piracicaba, São Paulo. As plantas foram conduzidas em recipientes de $500 \mathrm{~L}$, cheios de Latossolo Vermelho Amarelo, fase arenosa, denominado "Série Sertãozinho". Os tratamentos consistiram em manter 24 plantas com estresse hídrico (T0) e 24 plantas sem estresse hídrico (T1). A temperatura foliar foi obtida antes de cada irrigação, utilizando uma câmara térmica com imagens infravermelha. Com os dados de temperatura foliar e temperatura ambiente, calculou-se o índice de estresse hídrico da cultura (IEHC), em dois períodos aos 41 e 47 dias após o transplante (DAT), utilizando o limite basal inferior (LBI) teórico e calculado. As plantas submetidas ao estresse hídrico apresentaram maior temperatura foliar (Tc-Ta) do que aquelas sem déficit de irrigação. Os maiores valores do IEHC encontrados nos tratamentos não chegaram a 0,7. Independentemente, se o IEHC for calculado com o LBI teórico ou observado, as plantas sob estresse hídrico apresentam maiores IEHC em comparação com as plantas sem estresse hídrico. A identificação das plantas com estresse hídrico através do IEHC pode ser calculado tomando como referência o LBI teórico.
\end{abstract}

Palavras chave: temperatura foliar, manejo da irrigação, necessidade de irrigação.

\section{DETERMINATION OF CROP WATER STRESS INDEX FOR TOMATO CHERRY (Lycopersicum Solanum var. cerasiforme.) USING A THERMAL CAMERA}

\footnotetext{
${ }^{1}$ Engenheiro Agrônomo e Doutorando em PPGSA, ESALQ-USP. Professor Facultad de Agronomía, UCV, Maracay, Venezuela. ESALQ USP, Av. Pádua Dias 11. Piracicaba, SP. Fone +55 19 3447856. E-mail: ajfara@gmail.com.

${ }^{2}$ Prof. Dr. Associado, Depto. de Engenharia de Biossistemas, ESALQ-USP, Av. Pádua Dias 11. Piracicaba, SP.

3 Engenheira Agrícola e Doutoranda em PPGSA, ESALQ-USP. Professora Facultad de Agronomía, UCV, Maracay, Venezuela.

${ }^{4}$ Tecnólogo em Irrigação e Drenagem e Doutorando em PPGSA, ESALQ-USP. Prof. Instituto Federal Goiano, Morrinhos - GO.
} 


\begin{abstract}
Infrared thermometry is a possibility of detection of plant water stress by measuring the temperature of a portion of the canopy without requiring physical contact with the sheets. The purpose of this study was determining the crop water stress index in tomato cherry using the leaf temperature in plants as an indicator of water stress. Plants were grown in a greenhouse situated in the Department of Biosystems Engineering at the Escola Superior de Agricultura "Luiz de Queiroz "in Piracicaba, São Paulo. The plants were conducted in eight 500L boxes. The soil of the boxes is Oxisol eutrophic typical. Previously, were defined two groups: with induced water stress (T0; 24 plants) and without water stress (T1; 24 plants). The temperature of canopy was measured before irrigation using a thermal camera which captures infrared images. The leaf and air temperatures were used to calculation of crop water stress index (IEHC) in two moments: 41 and 47 days after transplanting (DAT) using the lower basal limit (LBI) and a theoretical method. The leaf temperature in group T0 was higher than observed in group T1. We found that: i) highest IEHC in both groups was less than 0.7; ii) IEHC based on theoretical method or LBI, showed that stressed plants have higher IEHC than plants with less water stress. The results indicate that water stress in tomato cherry could be estimated by IEHC based on LBI.
\end{abstract}

Keywords: leaf temperature, irrigation management, need for irrigation.

\section{INTRODUÇÃO}

Existe um grande interesse pelo desenvolvimento de pesquisas com a utilização mínima dos recursos hídricos disponíveis, visando o aumento da produção de alimentos com o uso eficiente da água. Neste contexto, o tomate é uma cultura irrigada, muito sensível ao estresse hídrico (WAISTER e HUDSON, 1970; SRINIVASA et al., 2001) e a maior sensibilidade ocorre no estágio de floração e formação dos frutos (HUANG, 1978).

Existem diferentes técnicas convencionais de detecção do estresse hídrico: sintomas visuais, balanço da água no solo, fluxo de seiva e a condutância estomática (Trentin, 2010). A termometria infravermelha é um método que possibilita a medição da temperatura de uma porção do dossel vegetativo, sem a necessidade de contato físico com as folhas. E a temperatura do dossel pode ser utilizada para quantificar o estresse hídrico na planta.

Existem diferentes modificações do índice que foi desenvolvido há 26 anos, chamado índice de estresse hídrico da cultura (IEHC), (CWSI, sigla em inglês), que é estabelecido com base empírica, adotado linhas de referência de temperatura.
Em uma cultura com estresse hídrico os estômatos permanecem fechados, a transpiração diminui o que ocasiona aumento da temperatura foliar. Quando uma planta está completamente sem estresse hídrico, a temperatura da folha é de 1 a $4{ }^{\circ} \mathrm{C}$ menor do que a temperatura ambiente; neste caso o IEHC é próximo de zero. Assim, quando a transpiração diminui, a temperatura da folha sobe e pode chegar de 4 a $6{ }^{\circ} \mathrm{C}$ acima da temperatura do ar. Neste caso, o déficit hídrico é alto, e a transpiração foliar é drasticamente reduzida e o IEHC fica próximo a 1 (JACKSON et al., 1982).

Segundo López-López et al. (2009) o método empírico de cálculo do índice de estresse hídrico foi desenvolvido por Idso et al. (1981) para quantificar o estresse hídrico de culturas em regiões áridas, onde a determinação depende de linhas de temperatura base sem e com estresse hídrico. As linhas de temperatura base são específicas de cada cultura e são influenciadas pelas condições climáticas (BUCKS et al., 1985). Jackson et al. (1981) modificou a IEHC incluindo: déficit pressão de vapor (DPV), saldo de radiação $(\mathrm{Rn})$ e resistência aerodinâmica (ra), para uma melhor previsão teórica dos efeitos do clima sobre a temperatura da cultura (KEENER; KIRCHER, 1983). 
O IEHC que sinaliza a necessidade de irrigação é específico da cultura e deve levar em consideração fatores como a resposta ao estresse hídrico, o valor provável da cultura e custo da água. Reginato e Howe (1985) constataram que a produção de algodão mostrou os primeiros sinais de declínio quando a média IEHC durante o cultivo foi maior do que 0,2 .

Assim, o objetivo deste estudo foi determinar o índice de estresse hídrico de tomateiros cereja através do comportamento da temperatura foliar das plantas, com e sem estresse hídrico, através de imagens infravermelhas.

\section{MATERIAL E MÉTODOS}

O ensaio para determinação do índice de estresse hídrico na cultura de tomate cereja (Solanum Lycopersicum var. cerasiforme.), com base em imagens infravermelhas foi conduzido durante o mês de outubro de 2014 (31 dias), em casa de vegetação, situada a $561 \mathrm{~m}$ de altitude, $22^{\circ} 42^{\prime} 41^{\prime \prime}$ de latitude sul e 47\%37'46” de longitude oeste, no Departamento de Engenharia de Biossistemas da Escola Superior de Agricultura "Luiz de Queiroz", em Piracicaba, São Paulo. A casa de vegetação possui altura de 3,0 m nas laterais e de 4,6 m na parte central, com cobertura de filme de polietileno de baixa densidade (PBDE), de 0,15 mm de espessura, tratado contra a ação de raios ultravioleta e com transparência de aproximadamente $80 \%$ e as laterais fechadas com sombrite $50 \%$.

O clima da região, segundo a classificação de Köppen (1948), é do tipo Cwa, subtropical úmido, com estiagem no inverno e verão quente e chuvoso. A temperatura média do mês mais quente é superior a $22^{\circ} \mathrm{C}$ e do mês mais frio inferior a $18^{\circ} \mathrm{C}$ (SENTELHAS, 1998).

As plantas foram conduzidas em oito caixas de $500 \mathrm{~L}$ cada. O solo das caixas é classificado como Latossolo Vermelho Amarelo, fase arenosa, denominado "Série Sertãozinho", apresentando: $19,24 \%$ de argila; 7,90\% silte; $72,86 \%$ de areia; densidade (Ds) de $1,26 \mathrm{~g} . \mathrm{cm}^{3}$; umidade com base em peso seco a capacidade de campo (CC) de $0,141 \quad \mathrm{~g} \cdot \mathrm{g}^{-1} \quad$ (umidade correspondente ao potencial mátrico $(\psi \mathrm{m})$ de 4,85 $\mathrm{kPa}$ ); umidade com base seco no ponto de murcha (PMP) de 0,070 g.g ${ }^{-1}$ (umidade correspondente ao $\psi \mathrm{m}$ de $1500 \mathrm{kPa}$ ); porosidade total do solo (PT) de 41,56\%; capacidade de agua disponível (CAD) de 18,2 $\mathrm{mm}$.

Foi definida uma lâmina de irrigação (para repor a $\mathrm{CAD}$ ), com uma frequência de dois dias para o período estabelecido. $\mathrm{O}$ cálculo da capacidade de água disponível (CAD) foi estimado usando os dados de física do solo, estabelecendo como potencial mátrico crítico $200 \mathrm{kPa}$, uma profundidade efetiva de $50 \mathrm{~cm}$, obteve-se uma lâmina de $21,1 \mathrm{~mm}$. Os tratamentos consistiram em manter 24 plantas com estresse hídrico (T0) e 24 plantas sem estresse hídrico (T1).

A reposição da lâmina de irrigação foi realizada a cada 2 dias, com base em volume, repondo o solo à capacidade de campo, até aos 36 dias após o transplante (DAT), quando a partir de então as plantas foram submetidas aos tratamentos propostos durante 31 dias.

O monitoramento dos dados climáticos (leituras a cada 30 segundos e médias a cada 15 minutos) foram realizados através uma estação meteorológica automática Campbell Sci., instalada na casa de vegetação, a uma altura de $2,0 \mathrm{~m}$ em relação ao solo que registrou a temperatura do ar $\left({ }^{\circ} \mathrm{C}\right)$ e umidade relativa do ar (\%) (sensor Vaissala HMP45C-L12 - Campbell Sci.). Os dados de radiação e velocidade do vento foram utilizados da estação meteorológica da LEB - ESALQ - USP - Piracicaba, SP, em função de a estufa haver ficado sem teto durante o período de aplicação dos tratamentos, embora a estação meteorológica interna tenha ficado em área coberta.

Foram tomadas fotografias com a Câmara Térmica (Térmica T640 da FLIR 307200 de pixels 640 x 480) aos 41, e 47 dias após transplantio (DAT), sendo as leituras realizadas antes das irrigações. Com os dados de temperaturas medidas nas plantas e temperatura do ambiente (Tmax e Tmin) medida pela estação 
meteorológica, correspondentes aos horários das leituras, calculou-se o índice IEHC por tratamento.

O índice de estresse hídrico da cultura (IEHC) foi calculado segundo Jackson et al. (1988) (Equação 01).

$$
I E H C=\frac{\left(T_{c}-T_{a}\right)-\left(T_{c}-T_{a}\right)_{L B I}}{\left(T_{c}-T_{a}\right)_{L B S}-\left(T_{c}-T_{a}\right)_{L B I}} \quad \text { Eq. }(01)
$$

Em que: Tc é a temperatura do dossel, Ta é a temperatura media do ar, $(\mathrm{Tc}-\mathrm{Ta})_{\mathrm{LBI}}$ é o linha base de temperatura inferior, correspondente à diferença de temperatura do ar para uma cultura bem regada quando a resistência para a perda de água é igual a zero ou correspondente a temperatura da superfície molhada; (Tc-Ta) $)_{\text {LBS }}$ é a linha base de temperatura superior correspondente à diferença de temperatura do ar quando a resistência para a perda de água do dossel aumenta sem limites ou correspondente à temperatura da superfície seca.

No caso da Tc, Meron et al. (2013) sugerem tirar os valores acima de $\mathrm{T}_{\mathrm{a}}+7^{\circ} \mathrm{C}$, porque se relacionam de forma segura com a temperatura do solo, enquanto descarta os objetos frios ajustando para um limite inferior a uma temperatura do ar abaixo de $10^{\circ} \mathrm{C}$. Então, $\mathrm{T}_{\mathrm{c}}$ fica entre um limite inferior de $\mathrm{T}_{\mathrm{a}}-10^{\circ} \mathrm{C}$ e um limite superior de $\mathrm{T}_{\mathrm{a}}+7^{\circ} \mathrm{C}$. No ensaio, foi estabelecido um limite superior de $\mathrm{T}_{\mathrm{a}}+10^{\circ} \mathrm{C}$.

Os cálculos das linhas de base de temperatura, inferior e superior, foram realizadas pelo método teórico, equação do Jackson et al. (1981) (Equação 02)

Assim, a linha base inferior $(\mathrm{Tc}-\mathrm{Ta})_{\mathrm{LBI}}$, foi estimada considerando a resistência estomática igual a zero, dossel completamente molhado, pela equação:

$$
(\mathrm{Tc}-\mathrm{Ta})_{\mathrm{LBI}}=\frac{\left(R_{n}-G\right) \cdot r_{a}}{\rho_{a r} C p\left(1+\frac{\Delta}{\gamma}\right)}-\frac{D V P}{\gamma\left(1+\frac{\Delta}{\gamma}\right)} \text { Eq. (02) }
$$

Em que: $r_{a}$ resistência aerodinâmica ou a resistência da camada limite para vapor de água $\left(\mathrm{s} . \mathrm{m}^{-1}\right), \gamma$, a constante psicrométrica $\left(\mathrm{kPa}^{\circ} \mathrm{C}^{-1}\right)$, $\mathrm{Rn}$, radiação liquida $\left(\mathrm{W} . \mathrm{m}^{-2}\right), \rho$ é a densidade do ar $\left(\mathrm{kg} . \mathrm{m}^{-3}\right), \mathrm{Cp}$ a capacidade de calor de ar
$\left(\mathrm{kJ} \cdot \mathrm{kg}^{-1} \cdot{ }^{\circ} \mathrm{C}^{-1}\right)=1,013, \Delta$ é a inclinação da curva que relaciona a pressão de vapor saturado à temperatura $\left(\mathrm{kPa} .{ }^{\circ} \mathrm{C}^{-1}\right)$, .

A resistência aerodinâmica $r_{a}\left(s . m^{-1}\right)$, foi determinada segundo Jackson et al (1988), sob condições neutras, a temperatura da superfície (essencialmente igual à temperatura do ar), ou seja, velocidade do vento $(\mathrm{u})>2 \mathrm{~m} . \mathrm{s}^{-1}$. No caso da velocidade do vento $(\mathrm{u}) \leq 2 \mathrm{~m} . \mathrm{s}^{-1}$, foi determinada segundo Thom e Oliver (1977) apud Jackson et al (1988). Sendo a constante psicrométrica $\gamma,\left(\mathrm{kPa}^{\circ} \mathrm{C}^{-1}\right)$, estabelecida segundo a Brunt (1952) citado por Allen et al. (1998); e o calor latente de vaporização, $\lambda$, Allen et al. (1998) citam a Harrison(1963); a densidade do ar $\rho_{\mathrm{ar}}\left(\mathrm{kg} \cdot \mathrm{m}^{-3}\right)$, segundo Allen et al. (1998). No caso da inclinação da curva que relaciona a pressão de vapor saturado à temperatura $(\Delta)$, utilizou-se a sugerida por Allen et al. (1998).

No caso da linha base superior (Tc Ta) ${ }_{\text {LBS }}$, que corresponderia ao uma superfície impermeabilizada, correspondente a temperatura da superfície seca, ou seja, uma resistência aproximando-se ao infinito, foi estimada pela equação 03:

$$
(\mathrm{Tc}-\mathrm{Ta})_{\mathrm{LBS}}=\frac{\left(R_{n}-G\right) \cdot r_{a}}{\rho_{a r} C p} \quad \text { Eq. (03) }
$$

Posteriormente, determinou-se o índice de estresse hídrico da cultura, substituindo na equação 1 à linha base inferior $(\mathrm{Tc}-\mathrm{Ta})_{\mathrm{LBI}}$ pela obtida como a mínima das diferenças entre a temperatura da cultura observada (Tc) e a temperatura média do ar (Ta); chamando neste caso índice de estresse hídrico da cultura observado (IEHC_Obs).

\section{RESULTADOS E DISCUSSÃO}

Durante o período de condução do experimento, a temperatura média do ar $\left({ }^{\circ} \mathrm{C}\right)$, variou entre os $32^{\circ}$ e $37^{\circ} \mathrm{C}$. Os valores médios diários das variáveis meteorológicas, tais como radiação solar global; radiação líquida; umidade relativa média; resistência aerodinâmica; déficit de pressão de vapor; temperatura média e velocidade do vento; podem ser verificados na (Tabela 1). 
Tabela 1. Valores médios das variáveis meteorológicas registradas no período experimental compreendido entre 41 e 47 DAT, outubro 2014.

\begin{tabular}{ccccccccc}
\hline DAT & $\begin{array}{c}\mathrm{Rs}^{1} \\
{\left[\mathrm{MJ} . \mathrm{m}-{ }^{2}\right]}\end{array}$ & $\begin{array}{c}\mathrm{Rn}^{2} \\
{\left[{\mathrm{MJ} . . \mathrm{m}^{-2]}}^{2}\right.}\end{array}$ & $\begin{array}{c}\mathrm{U}^{2} \mathrm{Rmed}^{3} \\
(\%)\end{array}$ & $\begin{array}{c}\mathrm{Ra}^{4} \\
{\left[\mathrm{~s} \mathrm{~m}^{-1}\right]}\end{array}$ & $\mathrm{DPV}$ & {$[\mathrm{kPa}]$} & $\begin{array}{c}\mathrm{Tmed}^{6} \\
{\left[{ }^{\circ} \mathrm{C}\right]}\end{array}$ & $\begin{array}{c}\mathrm{U}^{7} \\
{\left[\mathrm{~m}_{\mathrm{s}} \mathrm{s}^{-1}\right]}\end{array}$ \\
\hline 41 & 42,0 & 18,9 & 38,06 & 37,54 & 3,64 & 32,18 & 1,1 \\
47 & 82,2 & 45,2 & 53,34 & 35,46 & 4,04 & 36,55 & 1,3 \\
\hline
\end{tabular}

Radiação solar global; ${ }^{2}$ Radiação líquida; ${ }^{3}$ Umidade Relativa média; ${ }^{4}$ Resistencia aerodinâmica; ${ }^{5}$ Déficit de pressão de vapor; ${ }^{6}$ Temperatura média; ${ }^{7}$ Velocidade do vento.

As plantas submetidas a estresse hídrico (T0) apresentaram diferenças de temperaturas foliar (Tc - Ta), maiores do que as plantas do tratamento T1 (sem estresse hídrico). Os valores mínimos e máximos de temperatura da cultura (Tc - Ta), para plantas com e sem estresse hídrico foram de $-1,18^{\circ} \mathrm{C}$ a $9,71^{\circ} \mathrm{C}$ e de $-6,29^{\circ} \mathrm{C}$ a $-2,98^{\circ} \mathrm{C}$, respectivamente.

O índice de estresse hídrico da cultura (IEHC) calculado com o limite basal inferior teórico (LBI teórico), independentemente do tratamento, superestimou os valores do IEHC calculado com o limite basal inferior observado (LBI observado). Através da análise de regressão linear, observa-se que o índice de estresse hídrico da cultura (IEHC) calculado com o limite basal inferior observado (LBI observado) e o IEHC calculado com o limite basal inferior teórico (LBI teórico), apresentou valores de $\mathrm{R}^{2}$ de 0,86 e 0,74, para os tratamentos T0 e T1, respectivamente (Figura 1). Os elevados valores de $\mathrm{R}^{2}$ indicam, que nestas condições podem ser feitas as estimativas do IEHC com o limite basal inferior teórico (LBI teórico).

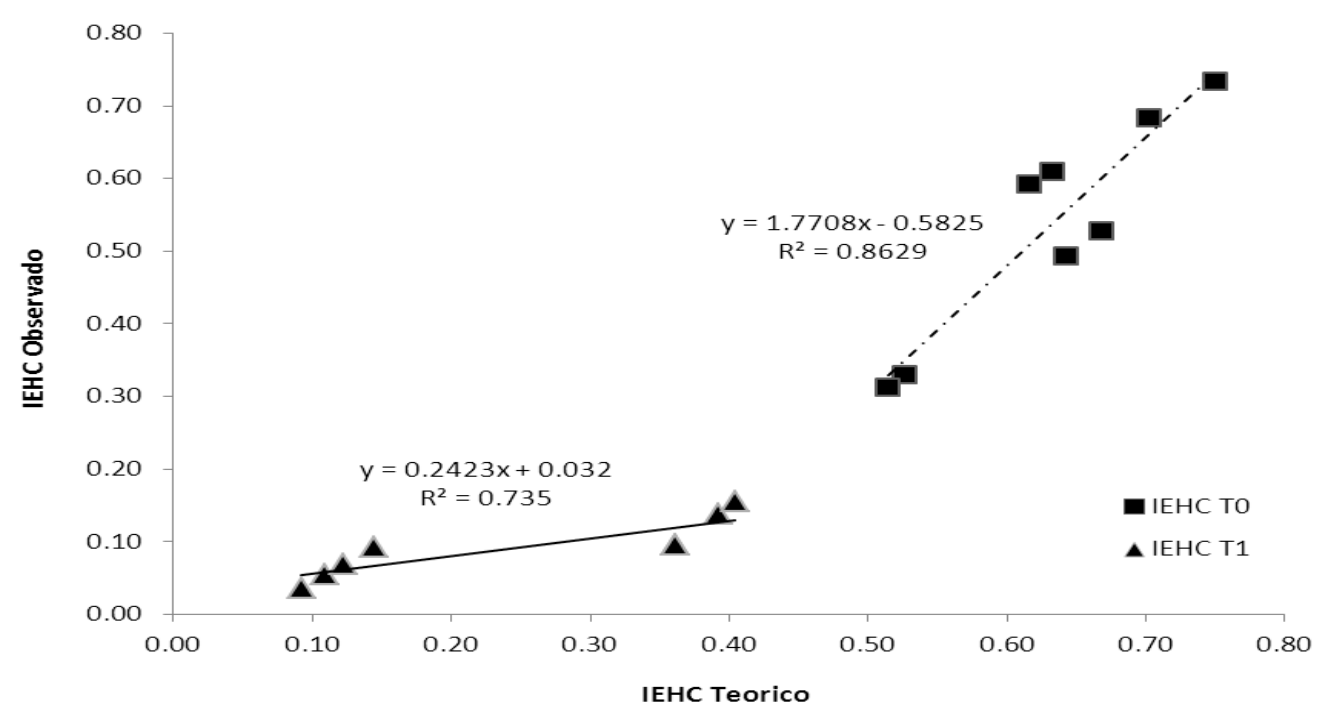

Figura 1. Relação entre o índice de estresse hídrico da cultura (IEHC) calculado com o limite basal inferior observado (LBS observado) e o IEHC calculado com o limite basal superior teórico (LBS teórico) para as plantas sem irrigação (IEHC T0) e plantas com irrigação (IEHC T1).

O menor ajuste $\left(\mathrm{R}^{2}=0,74\right)$ no tratamento T1 pode estar relacionado com algum mecanismo da cultura, em resposta ao aumento da temperatura (enrolamento de folha / alteração de ângulo foliar); questão não estudada pelo presente trabalho.

De forma geral, a variação do déficit de pressão de vapor (DPV), gera uma resposta variável nos tratamentos sem e com estresse hídrico. Com o aumento do déficit de pressão de vapor, há uma tendência do aumento do IEHC nas plantas submetidas a estresse hídrico e diminuição do IEHC nas plantas sem estresse hídrico. Entretanto, em ambos os casos os maiores valores de IEHC foram encontrados no tratamento T0 (estresse hídrico) (Figura 2), conforme também contatado por Trentin (2010) na cultura de cana de açúcar e Bezerra et al. (2014), na cultura do milho. 


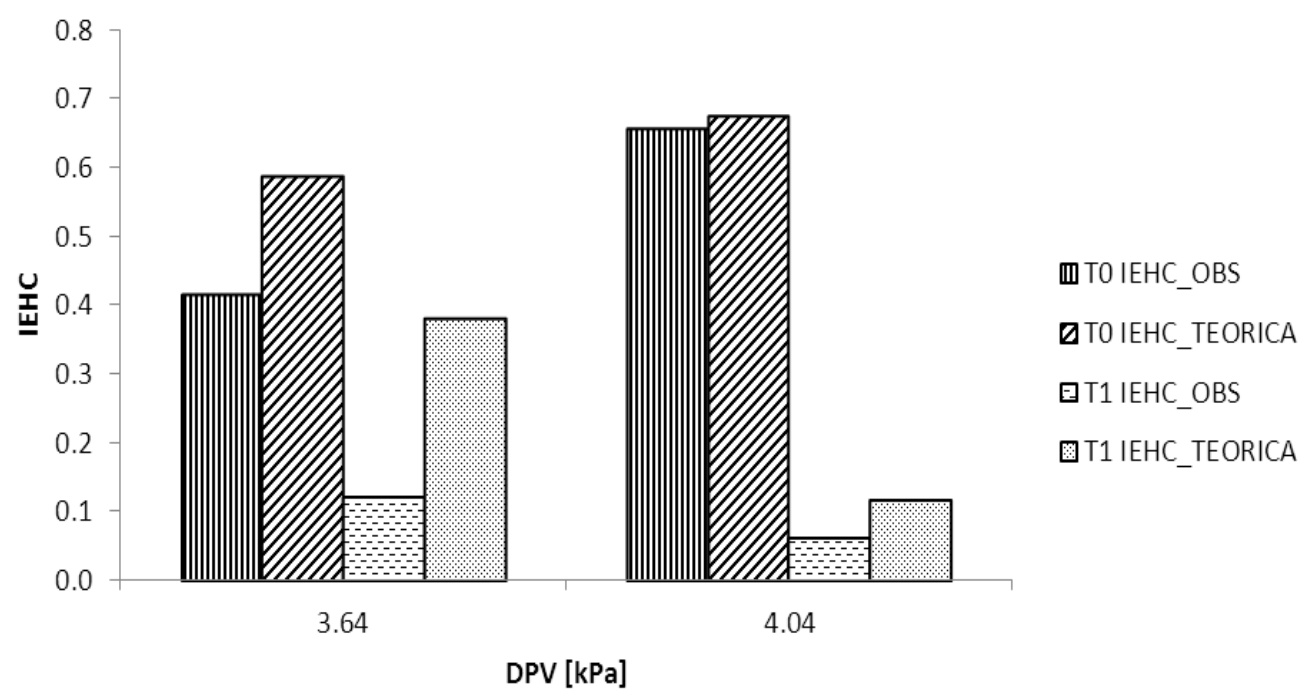

Figura 2. Relações entre o IEHC calculado pelo método teórico e o déficit da pressão de saturação do vapor d'água no ar (DPV), para a cultura do tomate cereja para T0 (sem irrigação) e T1 durante o ensaio usando a câmara infravermelha.

\section{CONCLUSÕES}

As plantas submetidas ao estresse hídrico apresentaram maior temperatura foliar (Tc-Ta) do que aquelas sem déficit de irrigação.

Independentemente se o IEHC for calculado com o LBI teórico ou o LBI observado, as plantas sob estresse hídrico apresentam maiores IEHC em comparação com as plantas sem estresse hídrico.

A identificação das plantas com estresse hídrico através do IEHC pode ser calculado tomando como referência o LBI teórico.

\section{AGRADECIMENTOS}

À Coordenação de Aperfeiçoamento de Pessoal de Nível Superior (CAPES), ao Conselho Nacional de Desenvolvimento Científico e Tecnológico $(\mathrm{CNPq})$, pelo financiamento das bolsas de estudos, à Fundação de Amparo a Pesquisa de São Paulo e à Empresa - Odebrecht, que através do Projeto PITE 2012/500837 disponibilizaram diversos equipamentos ao grupo de pesquisa em irrigação de cana-de-açúcar do Departamento de Engenharia de Biossistemas da ESALQ, alguns dos quais foram utilizados neste experimento.

\section{REFERENCIAS}

ALLEN, R. G.; PEREIRA, L. S.; RAES, D.; SMITH, M. Crop evapotranspiration: guidelines for computing crop water requirements. Rome: FAO, 1998. 300 p. (Irrigation and Drainage Paper, 56).

BEZERRA R. de S.; RODRIGUES T. R.; SILVEIRA, A. L. R. da; VELLAME, L. M.; CASAROLI, D; ARAÚJO. R. Estimativa de um índice de estresse hídrico para a cultura do milho com base na temperatura foliar. In: INOVAGRI INTERNATIONAL MEETING, 2014, Fortaleza. Anais eletrônicos... Fortaleza, INOVAGRI, 2014. Disponível em: http://dx.doi.org/10.12702/ii.inovagri.2014-a094 Acesso em 28 nov. 2014.

BRUNT, D. Physical and dinamical. Meteorology. Cambridge, Cambridge, Univ, Press., 1952, p. 428 pp.

BUCKS, D. A.; FRENCH, O. F.; NAKAYAMA, F. S.; FANGMEIER, D. D. Trickle irrigation management for grape production. Proceedings of the Third International Drip/Trickle Irrigation Congress, American Society of Agricultural Engineers, Fresno, CA (1985), pp. 204-211. 
HARRISON, L. P. Fundamentals concepts and definitions relating to humidity. In Wexler, A. (Ed.) Humidity and moisture. Vol 3, Reinhold Publishing Co., New York, NY, USA, 1963.

HUANG, W. C. Influence of soil moisture deficit at different stages of growth on the development yield, nutrients and water absorptions of tomato plants. Journal Agricultural China, v. 27, p.37-46, 1978.

IDSO, S. B.; JACKSON, R. D.; PINTER JR. P. J.; REGINATO, R. J.; HATFIELD, J. L. Normalizing the stress-degree-day parameter for environmental variability. Agricultural Meteorology, v. 24, p.45 - 55, 1981.

JACKSON, R. D.; IDSO, S. B.; REGINATO, R. J.; PINTER, P. J. Canopy temperature as a crop water stress indicator. Water Resources Research, v. 17, n. 4, p. 1133-1138, 1981.

JACKSON, R. D.; KUSTAS, W.P.; CHOUDHURY, B. J. A reexamination of the crop water stress index. Irrigation Science, v. 9, n. 4 , p. $309-317,1988$.

KEENER, M. E.; KIRCHER, P. L. The use of canopy temperature as an indicator of drought stress in humid regions. Agricultural Meteorology, v.28, n. 4, p. 339-349, 1983.

KÖPPEN, W. Climatología: con un estudio de los climas de la tierra. Fondo de Cultura Económica. México: 1948. 479 p.

LÓPEZ- LÓPEZ, R.; ARTEAGA-RAMÍREZ R.; VÁZQUEZ-PEÑA, M. A.; LÓPEZ-CRUZ, I. L.; SÁNCHEZ-COHEN, I.; RUIZ-GARCÍA, A. Índice de estrés hídrico del cultivo de tomate de cascara (Physalis ixocarpa Brot.) Revista Chapingo Serie Horticultura, v. 15, n. 3, p.
259-267, 2009

MERON, M.; SPRINTSIN, M.; TSIPRIS, J.; ALCHANATIS, V.; COHEN, Y. Foliage temperature extraction from thermal imagery for crop water stress determination. Precision Agriculture, v. 15, n. 5, p. 467-477, 2013.

REGINATO, R. J.; HOWE, J. Irrigation scheduling using crop indicators. Journal of Irrigation and Drainage Engineering, v. 111, n. 2, p. 125-133, 1997.

SENTELHAS, P. C. Estimativa diária da evapotranspiração de referência com dados de estação meteorológica convencional e automática. 1998. 97 p. Tese (Doutorado em Agronomia/Irrigação e Drenagem) - Escola Superior de Agricultura "Luiz de Queiroz", Universidade de São Paulo, Piracicaba.

SRINIVASA, N. K.; BHATT, R. M.; SADASHIVA, A. T. Tolerance to Water Stress in Tomato Cultivars. Photosynthetica, v. 38, n. 3, p. $465-467,2001$.

THOM, A. S.; OLIVER, H. R. On Penman's equation for estimating regional evaporation. Quarterly Journal of the Royal. Meteorological Society, v. 103, n. 436, p. $345-$ 357, 1997.

TRENTIN, R. Estimativa de um índice de estresse hídrico para a cultura da cana-deaçúcar com base na temperatura foliar. 2010. 81 p. Dissertação (Mestrado em Agronomia) Universidade Federal de Viçosa, Viçosa.

WAISTER, P. D.; HUDSON, J. P. Effect of soil moisture regimes on leaf deficit, transpiration and yield of tomatoes. Journal of Horticultural Science, v. 45, p. 359 - 370, 1970. 